\title{
Ephemeride des Planeten (393) Lampetia
}

gerechnet für $\mathbf{I}^{\mathrm{h}}$ M. Z. Berlin aus Elementen, die durch genäherten Anschluss der Jahrbuchselemente an die Heidelberger Beobachtung vom 10. März erhalten sind.

\begin{tabular}{|c|c|c|c|c|}
\hline 1902 & $\alpha$ & $\delta$ & $\log r$ & $\log \Delta$ \\
\hline März 30 & $11^{h} 5^{6^{m}} o^{s}$ & $-I^{\circ} 3^{8} \cdot 9$ & & \\
\hline April 3 & 5248 & $\begin{array}{ll}11 & 0.5\end{array}$ & $0.45^{8} 3$ & 0.2777 \\
\hline 7 & 4944 & 1021.0 & & \\
\hline II & I $146 \quad 51$ & -940.9 & 0.4543 & 0.2776 \\
\hline
\end{tabular}

\begin{tabular}{|c|c|c|c|c|}
\hline 1902 & $\alpha$ & $\delta$ & $\log r$ & $\log \Delta$ \\
\hline April I 5 & $11^{\mathrm{h}} 44^{\mathrm{m}} 13^{\mathrm{s}}$ & $-9^{\circ} \quad$ o: 9 & & \\
\hline 19 & 4151 & $\begin{array}{lll}8 & 21.3\end{array}$ & 0.4503 & 0.2814 \\
\hline 23 & $\begin{array}{ll}39 & 48\end{array}$ & $7 \quad 42.7$ & & \\
\hline 27 & 1138.6 & -7 & $0.446 I$ & 0.2887 \\
\hline
\end{tabular}

Berlin, Kgl. Recheninstitut, 1902 März 25.

Grösse I1.2.

A. Berberich.

\section{Planet 1902 HS.}

Aus Wien März I 3 und Königsberg März 16 ergiebt sich die Kreisbahn:

Epoche 1902 März I3.5 M. Z. Berlin.

$$
\begin{aligned}
& u=160^{\circ} 30^{\prime} 24^{\prime \prime}
\end{aligned}
$$

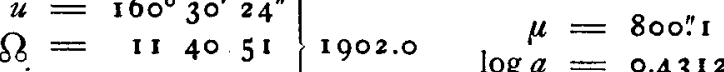

$$
\begin{aligned}
& i=44253\}^{2} \quad \log a=0.43124
\end{aligned}
$$

Ephemeride für $\mathbf{r}^{\mathrm{h}}$ M. Z. Berlin.

\begin{tabular}{|c|c|c|c|}
\hline 1902 & $\alpha$ app. & $\delta$ app. & $\log A$ \\
\hline März 27 & $\operatorname{II}^{\mathrm{h}} 22^{\mathrm{m}} 59^{\mathrm{s}}$ & $+6^{\circ}$ 1 $4: 6$ & $0.23^{8}$ \\
\hline 29 & 2124 & $\begin{array}{ll}6 & 20.3\end{array}$ & \\
\hline $3^{I}$ & I9 53 & $\begin{array}{ll}6 & 25.6\end{array}$ & \\
\hline April $\mathbf{2}$ & 1825 & $\begin{array}{ll}6 & 30.6\end{array}$ & \\
\hline 4 & I 7 I & 635.0 & 0.247 \\
\hline 6 & $\begin{array}{lll}\text { I I } & \text { I } 5 & 43\end{array}$ & +639.0 & \\
\hline
\end{tabular}

\begin{tabular}{|c|c|c|c|c|}
\hline & \multirow{2}{*}{$\frac{\alpha \text { app. }}{11^{h} \times 5^{m} 43^{s}}$} & \multirow{2}{*}{$\begin{array}{c}\delta \text { app. } \\
+6^{\circ} 39.0\end{array}$} & \multirow[t]{2}{*}{$\log A$} \\
\hline April & 6 & & & \\
\hline & 8 & 1429 & 642.6 & \\
\hline & Io & 1320 & $\begin{array}{ll}64.8 \\
6\end{array}$ & \\
\hline & I 2 & 1216 & $\begin{array}{ll}6 & 48.4\end{array}$ & 0.260 \\
\hline & I 4 & I I 18 & 650.3 & \\
\hline & I 6 & $\begin{array}{lll}11 & 10 & 25\end{array}$ & +651.7 & \\
\hline
\end{tabular}

Grösse 13.0 .

Die Beobachtung Heidelberg März 3 wird durch diese Bahn ziemlich gut dargestellt.

Der Planet ist wahrscheinlich identisch mit (359) [1893 M].

Berlin, Kgl. Recheninstitut, 1902 März 25.

Dr. P. V. Neugebauer.

\section{Einladung zur Astronomen-Versammlung in Göttingen.}

Der Vorstand der Astronomischen Gesellschaft beehrt sich, die Herren Mitglieder zu der statutenmässigen Versammlung, welche nach Beschluss der letzten Versammlung in Göttingen stattinden soll, einzuladen. Die Versammlung ist auf die Tage von Montag den 4. bis Donnerstag den 7. August 1902

anberaumt. Die Herren Mitglieder werden ersucht, sich nach ihrer Ankunft in Göttingen in der dortigen Sternwarte zu melden, um nähere Mittheilungen in Empfang zu nehmen.

Anträge oder Mittheilungen, welche die Herren Mitglieder auf der Versammlung an die Gesellschaft zu richten beabsichtigen, sind nach $\S 27$ der Statuten vorher bei dem Vorstande einzureichen. Dieser wird einige Tage vor der Eröffnung der Versammlung in Göttingen zusammentreten.

München, Berlin, Potsdam, 14. März 1902.

$H$. Seeliger, Vorsitzender.

R. Lehmann-Filhés, G. Müller, Schriftfübrer.

Inhalt zu Nr. 3781-82. A. Abetti. Asteroidi osservati ad Arcetri nel Igor. I93. - H. Struve. Beobachtungen von kleinen Planeten. 21 r. S. D. Tozenley. Filar-micrometer observations of asteroids. 213. - M. Wolf. Photographische Beobachtungen von kleinen Planeten. 215. - M. Wolf. Photographische Beobachtungen von kleinen Planeten. 217. - L. F. Gruey. Observations de la planète r 902 HT. 217. - Th. Brédikhine. Ueber den Schweif des Cometen Igor I. 219. - C. Koss. Beobachtung der Sonnenfinsterniss Igor Nov. Io. 219 . - R. H. Tucker. Meridian Circle Observations of Comparison Stars for the Major Planets. 219. - W. Ceraski. Découverte d'une variable 4.1902 Geminorum. 22 r. - T. Köhl. Aufforderung betr. Zeichnungen von Meteoren. 221. - A. Berberich. Ephemeride des Planeten (393) Lampetia. $223 .-P$. $V$. Neugebauer. Planet 1902 HS, 223. - Einladung zur Astronomen-Versammlung in Göttingen. 223. 